\title{
La technologie au service de la formation pratique : la captation vidéo de manipulations et d'interventions chirurgicales dans le cadre d'une technique en santé animale
}

\author{
Auteures \\ Émilie St-Pierre, doctorante, Université du Québec à Chicoutimi, Canada, \\ emilie.st-pierre1@uqac.ca \\ Valérie Deschenes, médecin vétérinaire, cégep de Saint-Félicien, \\ Canada, \\ vdeschenes@cegepstfe.ca \\ Josée Duranleau, médecin vétérinaire, cégep de Saint-Félicien, Canada, \\ iduranleau@cegepstfe.ca
}




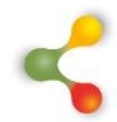

\section{REVUE HYBRIDE DE L'ÉDUCATION}

\section{Résumé}

La pandémie de la COVID-19 a forcé la fermeture de nombreux établissements scolaires (OCDE, 2020). Alors que l'enseignement à distance a permis la continuité des apprentissages académiques, il en a été autrement pour la continuité des enseignements pratiques (OCDE, 2020). Cet article présente le point de vue d'une conseillère pédagogique et d'une enseignante en technique en santé animale du cégep de SaintFélicien relativement à l'utilisation d'un système de captation vidéo pour le maintien des apprentissages pratiques à distance. L'article situe d'abord le contexte ayant mené à l'installation dudit système avant d'aborder l'utilité de celui-ci dans le cadre de l'enseignement pratique.

Mots-clés : enseignement collégial; enseignement à distance; enseignement pratique; technique en santé animale; captation vidéo 


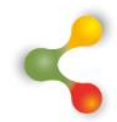

\section{REVUE HYBRIDE DE L'ÉDUCATION}

\section{Le maintien des enseignements pratiques en contexte pandémique : une mise en contexte}

II y a déjà un an, soit en mars 2020, le contexte pandémique engendré par la COVID-19 venait chambouler nos vies de façon drastique en obligeant la population québécoise à vivre une importante réorganisation sociale caractérisée par des efforts d'isolement et de distanciation physique (Leblanc et al., 2020 ; Ng et Badets, 2020). Dans la foulée, le domaine de l'éducation n'a pas été épargné alors que les mesures sanitaires imposées par le gouvernement ont forcé l'interruption de la scolarisation traditionnelle (OCDE, 2020).

Cette situation exceptionnelle a forcé la fermeture de nombreux établissements scolaires dans 188 pays, touchant du même coup plus de 1,7 milliard de jeunes et leur famille (OCDE, 2020). Malgré les efforts considérables déployés par la communauté éducative, les étudiants ont dû recourir à leurs ressources personnelles afin de poursuivre leurs apprentissages en ligne. Alors que l'enseignement à distance a permis une certaine continuité des apprentissages académiques, les mesures sanitaires ont davantage complexifié la continuité des enseignements pratiques (OCDE, 2020).

Malgré tout, la débrouillardise et la mobilisation de certains enseignants ont permis la mise en place de stratégies innovantes permettant de poursuivre, dans la mesure du possible, la formation pratique de leurs étudiants. C'est le cas de mesdames Valérie Deschenes et Josée Duranleau, toutes deux vétérinaires de formation. Elles ont pratiqué la médecine vétérinaire pendant plusieurs années avant de se tourner vers l'enseignement collégial. $\mathrm{D}^{\text {re }}$ Deschenes, qui a travaillé pendant plus de 12 ans comme enseignante à la technique en santé animale dans différents établissements d'enseignement collégial, occupe, depuis peu, le poste de conseillère pédagogique à l'intégration des technologies de l'information et de la communication (TIC) au cégep de Saint-Félicien. Celle-ci avait préalablement obtenu une attestation d'études collégiales (AEC) en développement web. De son côté, $D^{r e}$ Duranleau a exercé la médecine vétérinaire auprès de petits animaux pendant plus de 12 ans. Depuis, elle a piloté différents cours à la technique en santé animale au cégep de SaintFélicien. Elle se spécialise dans la formation de futurs techniciens en santé animale souhaitant travailler en centre de recherche.

Elles ont été rencontrées expressément afin de les questionner sur le maintien de la formation pratique dans le cadre de la technique en santé animale dispensée au cégep de Saint-Félicien et, plus précisément, relativement à l'utilisation d'un système de caméra pour assurer la captation vidéo de leurs interventions chirurgicales dans une optique de formation. Cet article se veut un témoignage pour les enseignants qui désireraient en apprendre davantage relativement aux pratiques 


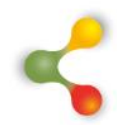

\section{REVUE HYBRIDE DE L'ÉDUCATION}

innovantes permettant la continuité des apprentissages pratiques en contexte d'enseignement en ligne.

Une approche de proximité en présentiel comme à distance : une nécessité pour la formation pratique

Tant pour $D^{\text {re }}$ Deschenes que pour $D^{\text {re }}$ Duranleau, il demeure primordial de favoriser le développement de l'autonomie auprès des étudiants de la technique en santé animale afin de former de futurs techniciens compétents et pour faciliter leur intégration sur le marché du travail. Dans cette optique, elles préconisent l'enseignement de méthodes de travail diversifiées pour préparer les étudiants à intégrer des milieux de travail variés (cliniques vétérinaires de quartier, clinique vétérinaire de référence, milieux de recherche). Elles préconisent également toutes deux une approche humaine de l'enseignement en priorisant le développement d'une relation de proximité avec leurs étudiants. $D^{\text {re }}$ Deschenes souligne à cet égard que le fait de développer une relation positive avec eux facilite la gestion de classe et la discussion avec ses étudiants.

Dans cette optique, celles-ci utilisaient, bien avant la fermeture des écoles engendrée par le contexte pandémique, diverses plateformes numériques à des fins de communication et de formation. Notamment, la plateforme Moodle leur offrait d'ores et déjà la possibilité de déposer des documents théoriques, mais également des capsules vidéo leur permettant d'enseigner certaines notions anatomiques, des manipulations ou des interventions chirurgicales de façon explicite. Cela permettait aussi aux étudiants de mieux se préparer et de bénéficier du temps de laboratoire qui leur est alloué et de diminuer la manipulation des animaux. Cela permettait alors aux étudiants de suivre les cours à leur rythme et de revenir, en cas de besoins, sur certaines notions plus spécifiques en laboratoire. Également, toutes deux utilisaient le portail Col.net, lequel permettait la remise des résultats académiques à leurs étudiants.

Outre l'utilisation de plateformes éducatives, $D^{\text {re }}$ Deschenes privilégiait la création de groupes de discussion avec tous ses étudiants afin de rester en contact avec eux et maintenir une certaine proximité. Cette dernière mentionne d'ailleurs l'appréciation et l'aisance avec lesquelles ses étudiants utilisent ce moyen de communication dans le cadre de ses cours en soulignant que ceux-ci ont même, à certains moments, pris l'initiative de créer un groupe de discussion.

\section{La proactivité et la créativité comme mots d'ordre!}

La proactivité et la créativité comme mots d'ordre : c'est ce que le discours des docteurs Deschenes et Duranleau laisse transparaitre. D ${ }^{\text {re }}$ Deschenes souligne, pour sa part, qu'elle avait dû «prévoir le coup » en faisant référence à la fermeture des écoles et au mode de prestation des cours à distance. En effet, Dre Deschenes mentionne avec un certain 


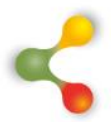

\section{REVUE HYBRIDE DE L'ÉDUCATION}

soulagement qu'elle avait pris la décision, au mois de décembre précédant la fermeture des écoles, d'adapter son matériel pédagogique afin d'offrir ses cours en ligne. Ainsi, au moment de l'annonce des mesures de confinement lors de la première vague de la pandémie, une partie du travail nécessaire à la poursuite de l'enseignement en ligne (adaptation du matériel et des méthodes d'évaluation) était entamée pour $D^{\text {re }}$ Deschenes. Cette dernière fait d'ailleurs preuve d'une aisance avec l'utilisation des technologies dans un contexte éducatif. Pour elle, la souplesse que permet l'enseignement en ligne relativement aux possibilités d'adapter le contenu au rythme des étudiants est un atout majeur, et ce, principalement dans les cours où la matière est dense et complexe. Elle dispensait déjà son cours en alternance entre une formule synchrone (une fois par semaine) et asynchrone.

Malgré les possibilités offertes par les plateformes numériques, l'enseignement pratique tel que l'enseignement des méthodes d'interventions chirurgicales et la démonstration de manipulations très précises effectuées sur de petits animaux demeuraient un défi pour les docteures Deschenes et Duranleau. En effet, lors de la fermeture des écoles, le cégep de Saint-Félicien a dû relocaliser les animaux qui étaient présents au sein de ses locaux et qui permettaient aux étudiants de la technique en santé animale de mettre en pratique les notions vues dans leurs cours. De plus, les étudiants étant à distance, il n'aurait, de toute façon, pas été possible pour eux de les manipuler. Tous les enseignants du département, dont mesdames Deschenes et Duranleau, ont dû être proactifs et faire preuve de créativité afin de pallier cette lacune. Bien que les capsules vidéo descriptives déposées sur la plateforme Moodle étaient relativement efficaces sur le plan théorique, elles ne permettaient pas de répondre aux standards de formation : encore fallait-il pouvoir mettre les étudiants en action pour qu'ils deviennent des techniciens compétents et autonomes. Les docteures Deschenes et Duranleau ont été proactives sur le plan de l'utilisation des technologies de l'information et de la communication (TIC) avant la pandémie puisqu'elles avaient déjà développé des compétences relatives à leur utilisation. La survenue d'une cyberattaque en septembre 2020 , soit quelques mois avant le début de la pandémie ${ }^{1}$, avait tout de même précipité l'utilisation de certains logiciels tels que Microsoft 365 avec lequel elles étaient désormais plus familières.

À titre d'exemple, Dre Duranleau explique qu'elle a demandé à ses étudiants de se confectionner, à la maison et avec le matériel qu'ils avaient sous la main, des peluches représentant de petits animaux (ex.: des souris). $D^{\text {re }}$ Duranleau offrait alors, et ce, pendant plus de quatre semaines consécutives, des rencontres individuelles sur la plateforme Zoom avec tous ses étudiants afin de leur présenter certaines manipulations et notions

\footnotetext{
1 Le 17 septembre 2019, le cégep de Saint-Félicien a été victime d'une cyberattaque entrainant un important vol de données.

http://cegepstfe.ca/fr/nouvelle/le-cegep-de-st-felicien-victime-une-cyberattaque
} 


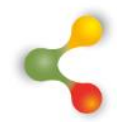

\section{REVUE HYBRIDE DE L'ÉDUCATION}

anatomiques. De cette façon, ses étudiants pouvaient manipuler des « animaux » et se mettre en action. Cette dernière met toutefois en garde les enseignants qui souhaiteraient reproduire cette façon de faire en mentionnant que cela lui a demandé une quantité d'énergie phénoménale.

\section{La captation vidéo des manipulations et interventions chirurgicales comme solution prometteuse pour la formation pratique à distance}

Malgré les efforts déployés, certains problèmes persistaient. D'une part, les étudiants qui auraient dû suivre le cours d'anesthésie n'avaient pas pu expérimenter la manipulation sur de vrais animaux en raison du confinement. Or, les cours de chirurgie dispensés par madame Deschenes, offerts après ceux sur l'anesthésie pour des raisons logiques, nécessitent une bonne maitrise des habiletés apprises dans le premier cours. Afin de pallier cette lacune, un programme de rattrapage impliquant la libération d'enseignants et des cours supplémentaires pour enseigner les techniques aux étudiants a été mis en place entre la première et la seconde vague de la pandémie.

D'autre part, les cours de chirurgie dispensés par $D^{\text {re }}$ Deschenes ne peuvent se donner à distance et les animaux nécessitant des chirurgies doivent recevoir les traitements appropriés qu'il y ait présence d'étudiants ou non. Également, l'étroitesse de la salle de chirurgie faisait en sorte qu'il était impossible que les étudiants y soient présents considérant les consignes de distanciation. Par ailleurs, dans le cadre de ses cours, $D^{\text {re }}$ Duranleau doit manipuler de petits animaux et il devient parfois complexe pour les étudiants (même en présentiel) de voir adéquatement les manipulations effectuées pour ensuite les reproduire. Devant cette situation, les enseignants du département ont redoublé d'efforts et de créativité afin de trouver une solution qui permettrait aux étudiants d'assister aux interventions depuis le confort de leur foyer. La captation vidéo des interventions apparaissait alors comme une solution prometteuse.

Selon les docteures Duranleau et Deschenes, la mise en place de deux caméras permet une captation suffisamment claire des interventions et des manipulations afin de que les étudiants puissent avoir une bonne vision de l'intervention. En effet, alors que l'une des caméras est fixée au mur et permet d'avoir une vision plus large du laboratoire, l'autre, attachée sur une lampe chirurgicale, facilite une certaine mobilité et offre une vue en plongée du plan de travail. À cet effet, $D^{\text {re }}$ Duranleau soutient qu'elle a ellemême pu expérimenter la qualité de ce système de caméra alors qu'elle s'est retrouvée en isolement à la maison et qu'aucun autre enseignant n'était en mesure d'enseigner le contenu de son cours. Se trouvant dans cette situation, $D^{\text {re }}$ Duranleau a alors eu l'idée de demander à ses étudiants de lui faire la démonstration de certaines manipulations devant la caméra. Ainsi, cela lui permettait d'exercer son rôle, et ce, même depuis le confort de sa maison. Elle souligne d'ailleurs son étonnement face à la qualité de 


\section{$\&$}

\section{REVUE HYBRIDE DE L'ÉDUCATION}

la vidéo qui la faisait se sentir «comme si elle était face à eux ». Elle spécifie à ce propos que l'utilisation d'écouteurs et de micro participait largement à cette sensation en permettant d'atténuer le bruit ambiant.

Bien que le système de caméras ne permette pas aux étudiants de faire les manipulations comme s'ils étaient en classe, $D^{\text {re }}$ Deschenes mentionne qu'il leur permet d'assister visuellement aux laboratoires ne serait-ce que pour voir le déroulement des interventions chirurgicales, les mouvements et les déplacements des membres de l'équipe présente en salle de chirurgie, la façon dont les manipulations se coordonnent et les outils nécessaires pour la réalisation d'une intervention spécifique. Elle précise également qu'il importe de connaitre adéquatement le protocole nécessaire à la réalisation des interventions afin d'assurer leur bon déroulement et d'établir une bonne coordination entre les membres de l'équipe. Par ses propos, madame Deschenes met en exergue l'importance de bien comprendre le protocole et les raisons justificatives pour lesquelles celui-ci doit être fait d'une façon précise et non d'une autre, afin de former de futurs techniciens en santé animale compétents et aptes à porter un regard critique sur leur pratique. Dans le même ordre d'idées, Dre Duranleau souligne qu'une bonne connaissance des procédures et de leur justificatif représente une valeur ajoutée pour le développement de l'autonomie des futurs techniciens en santé animale.

Enfin, selon Dre Duranleau, un autre atout de la mise en place de ce système de caméras réside également dans le fait que celui-ci permet d'accommoder les étudiants internationaux ou les étudiants qui doivent s'absenter de classe, et ce, en contexte de pandémie ou non. Cette dernière perçoit donc une utilité à cette installation au-delà du contexte pandémique. Pour illustrer ses propos, celle-ci présente l'exemple de l'une de ses étudiantes internationales qui désirerait aller voir sa famille pour le congé des Fêtes. Cela impliquait également qu'elle doive être placée en isolement à son retour en région et qu'elle doive, en conséquence, s'isoler pendant deux semaines. Cet isolement l'empêcherait de se présenter à son cours intensif. Le système de caméra pourrait dès lors permettre à cette étudiante d'assister, du moins virtuellement, à ses cours pratiques malgré son isolement préventif, ce qui lui éviterait de prendre du retard sur les notions vues en classe. Sans ce système de caméra, l'étudiante en question aurait probablement préféré ne pas retourner dans sa famille pendant les Fêtes, car elle aurait eu une trop grande quantité de matière à rattraper.

\section{La captation vidéo des manipulations et interventions chirurgicales : quels défis se posent?}

Bien que l'utilisation de caméras en contexte de formation pratique à distance, notamment dans le cadre d'une technique en santé animale, permette une certaine continuité des apprentissages, elle présente tout de 


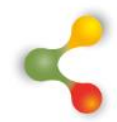

\section{REVUE HYBRIDE DE L'ÉDUCATION}

même certains défis. À cet effet, Dre Duranleau mentionne que l'incertitude relative à la survenue de problèmes techniques lui occasionne un certain stress, et ce, même en ce qui a trait à l'utilisation de la plateforme Moodle. $D^{\text {re }}$ Deschenes ajoute, en réponse au commentaire de sa collègue, que «le jeu en vaut malgré tout la chandelle ", considérant tous les avantages relatifs à l'utilisation de la technologie dans l'enseignement à distance et considérant que, dans la plupart des cas, cela fonctionne adéquatement. Elle ajoute à ce propos qu'il vaut mieux pour un étudiant d'être en mesure de visualiser une intervention de manière virtuelle que de ne pas pouvoir la visualiser du tout.

Également, Dre Duranleau mentionne que l'utilisation même des caméras pouvait lui causer un stress important puisqu'elle n'avait jamais eu à utiliser un système similaire par le passé. Cependant, l'utilisation fréquente du système de caméras lui a apporté une certaine confiance, non seulement envers le système lui-même, mais également envers ses compétences par rapport à leur utilisation. Elle se dit même ravie d'avoir accès à ce type de système.

\section{Voir plus loin que la pandémie...}

Au-delà du contexte pandémique et de l'enseignement à distance, $D^{\text {re }}$ Deschenes souligne que l'utilisation du système de caméras permet le partage de matériel pédagogique avec les autres classes. Pour appuyer ses propos, celle-ci mentionne, à titre d'exemple, le fait qu'une situation exceptionnelle ou très peu commune puisse survenir lors d'une chirurgie. Dans le cas où cette intervention serait filmée grâce au système de caméras mis en place, cela pourrait permettre de partager les images de ladite intervention à d'autres groupes classes, bonifiant alors la formation de ces derniers. Dre Deschenes renchérit en affirmant que c'est d'ailleurs l'argument de la pérennité des installations et de la possible bonification de la formation qui a fait en sorte que la direction de l'établissement d'enseignement collégial a adhéré à l'idée de mettre en place le système de caméras.

$D^{\text {re }}$ Duranleau mentionne également que l'utilisation de capsules vidéo éducatives était déjà une pratique répandue auprès des enseignants du département et que les étudiants avaient déjà accès à une page Moodle sur laquelle ils pouvaient retrouver tous les vidéos et outils que les enseignants avaient partagés au cours de leur formation collégiale. Ainsi, l'installation d'un système plus performant et offrant deux angles de captation est, selon cette dernière, un atout indéniable sur le long terme. Cela permet la production de vidéos de meilleure qualité grâce auxquelles les étudiants pourront bénéficier d'une meilleure vision des manipulations réalisées pour ensuite les reproduire dans le cadre de leurs cours ou, ultérieurement, de leur emploi. Également, $D^{\text {re }}$ Deschenes mentionne qu'avant l'installation du système de caméras, la réalisation de capsules vidéo éducatives nécessitait le soutien de l'équipe de l'audiovisuel du 


\section{$\&$}

\section{REVUE HYBRIDE DE L'ÉDUCATION}

cégep. Or, depuis l'installation dudit système, $\mathrm{D}^{\text {re }}$ Deschenes remarque, en ce qui la concerne, une plus grande facilité à produire les vidéos de façon autonome, ce qu'elles apprécient toutes deux énormément.

En plus des multiples avantages liés à la formation pratique à distance, $D^{\text {re }}$ Duranleau mentionne que la flexibilité qu'offre ce système de caméras la libère d'un stress important, ce à quoi acquiesce $D^{\text {re }}$ Deschenes. En effet, $D^{r e}$ Duranleau souligne que le système de captation vidéo lui permet une plus grande marge de manœuvre et plus de possibilités dans les cas où ses étudiants ou elle-même doivent d'absenter. Elle mentionne également qu'elles n'ont désormais plus à prendre rendezvous avec un membre de l'équipe de l'audiovisuel pour la réalisation des capsules, ce qui était le cas auparavant. Ainsi, cela fait en sorte qu'elles sont davantage libres de le faire au moment qui leur semble le plus opportun, tout en ayant la possibilité d'avoir plus d'une prise de vue.

\section{Des conditions primordiales à la mise en place d'un tel système}

Interrogée sur les conditions primordiales à la mise en place d'un tel système, $D^{\text {re }}$ Duranleau souligne d'abord l'importance d'établir une étroite collaboration entre la direction des études et les membres du personnel enseignant. Plus précisément, elle mentionne l'importance de consulter les enseignants afin de connaitre leurs besoins relatifs à l'utilisation du système de caméras. À ce propos, Dre Deschenes précise qu'il est essentiel que les enseignants du département aient une vision commune de leur enseignement. Selon elle, le tout facilite la formation des étudiants en assurant une certaine continuité d'un cours à l'autre. L'installation du système de caméras s'inscrit dans cette vision commune qu'ont les enseignants du département.

Dre Duranleau mentionne également que les enseignants doivent faire preuve de disponibilité et avoir de bonnes capacités d'adaptation. En effet, au moment de l'installation du système de caméras, certains enseignants ont dû prendre de leur temps personnel durant l'été pour aller au cégep et accompagner les techniciens chargés de l'installation et de la programmation du système de caméras. Puisqu'ils sont les utilisateurs principaux du système de caméras, leur aide a été sollicitée afin de pister les installateurs sur l'endroit le plus approprié pour installer les caméras. De plus, les enseignants du département ont dû s'adapter en modifiant l'horaire de leur cours afin de libérer le laboratoire pendant une semaine pour permettre l'installation du système.

\section{Que peut-on en conclure?}

En conclusion, les docteures Duranleau et Deschenes affirment que les quelques défis relatifs à l'utilisation du système de caméras sont minimes lorsqu'ils sont comparés aux avantages de son utilisation. Elles terminent d'ailleurs l'entretien en mentionnant qu'elles ne perçoivent pas 


\section{$\varepsilon$}

\section{REVUE HYBRIDE DE L'ÉDUCATION}

de réelles embûches concernant l'utilisation du système et qu'elles sont optimistes quant à l'identification de retombées positives supplémentaires en ce qui a trait à leur tâche d'enseignement, mais également pour la bonification de la formation de leurs étudiants. En effet, Dre Deschenes souligne qu'étant donné la récence de l'installation du système de caméras, celui-ci n'a pas été utilisé à son plein potentiel. Elle se dit confiante envers les multiples utilités de celui-ci qu'il reste à découvrir et qui pourront, éventuellement, contribuer à faciliter son travail et bonifier la formation de ses étudiants. $D^{\text {re }}$ Duranleau abonde dans le même sens en mentionnant que le système de caméras pourra éventuellement faciliter la gestion de classe en permettant de voir certains étudiants effectuant une manipulation quelconque, mais qui ne se retrouvent pas dans le même local que leur enseignant. Ainsi, cela devrait lui permettre de rectifier plus rapidement les pratiques des étudiants en cas de manipulations inappropriées. 


\section{REVUE HYBRIDE DE L'ÉDUCATION}

\section{Références}

$\mathrm{Ng}$, E. et Badets, N. (2020). Les impacts de la Covid-19 : Le bien-être des enfants au Canada. Institut Vanier de la famille. https://institutvanier.ca/l-impact-de-la-covid-19-le-bien-etre-desjeunes-au-canada/

Organisation de coopération et de développement économiques. (2020). Regards sur l'éducation 2020 : les indicateurs de l'OCDE. OCDE. https://www.oecd-ilibrary.org/docserver/7adde83a-

fr.pdf?expires $=1614186531 \& \mathrm{id}=\mathrm{id} \&$ accname $=$ guest $\&$ checksum $=\mathrm{C}$ F32103B3C13D6B4F5A512CE8A9474EC 